\title{
Cohesion of bird nests
}

\author{
Ignacio Andrade-Silva ${ }^{1, *}$, Théo Godefroy ${ }^{1, * *}$, Olivier Pouliquen ${ }^{1, * * *}$, and Joel Marthelot ${ }^{1, * * * *}$ \\ ${ }^{1}$ Aix-Marseille University, CNRS, IUSTI (Institut Universitaire des Systémes Thermiques Industriels), 13013 Marseille, France.
}

\begin{abstract}
One striking difference between aggregates of flexible frictional fibres and other granular materials like rigid spheres is the effective cohesion of their assembly. While glue or capillary bridges are needed to shape aggregates of spherical particles and build sandcastles, for fibres, no need for glue to build a nest. Here we study an assembly of mono disperse flexible fibres. We first use X-ray microtomography to characterise the geometry of the initial assembly, the number of contact points and mean curvatures of the fibres. Using forcedisplacement measurements, we characterise the macroscopic cohesive strength of the aggregate by varying the geometry of the fibres, the fibres mechanicals properties and the packing of the preparation. Finally, we relate the macroscopic mechanical behaviour of the assembly with the filament reorganisation at the microscopic scale.
\end{abstract}

\section{Introduction}

Many bird species rely on no other ingredients than the elasticity of sticks and the frictional contacts between them to build their nests [1]. Most of bird nests can be seen as networks of randomly packed entangled fibres which conform mechanically stable structures that are capable of bear the weight of the bird and its eggs. In addition, this structure is cohesive without the need of some cohesive ingredient like glue or water capillary bridges. This effective cohesion is a result of frictional fibre-fibre forces whose magnitudes depend on the bending state of the fibres. To achieve such effective cohesion, the right choice of the aspect ratio of the particles and their right mechanical properties seems crucial [2]. Although considerably progress has been made in the past in understanding the mechanical response of fibre-based materials under compression [3], almost no studies focus on a direct characterisation of their cohesive strength.

Here we study an assembly of monodisperse flexible fibres. We first use X-ray microtomography to characterise the geometry of the initial assembly, the number of contact points and mean curvatures of fibre aggregate at different degree of precompression. Then, we establish an experimental protocol that allows us to measure the cohesive strength of the aggregate as the resistive force against traction.

\section{Sample preparation and statistical characterisation}

\subsection{Sample preparation}

We first consider a cylindrical assembly of monodisperse plastic fibres (Plastinyl 6.6) of length $L=5.5 \mathrm{~cm}$ and diameter $d=0.4 \mathrm{~mm}$ (aspect ratio of 137.5). The fibres have Young modulus $E$ of approximately $3 \mathrm{GPa}$ and density $\rho=1056 \mathrm{~kg} \mathrm{~m}^{-3}$. The cylindrical assembly is built by rain filling the fibres under gravity in a silo of diameter $12.5 \mathrm{~cm}$. Our samples contain typically 7000 fibres. To obtain an orientational distribution as random as possible the fibres pass through two grids of elastic strings before falling into the container. After the container is filled, the walls are carefully removed, obtaining a stable tower of fibres as shown in Fig. 1(a). Our samples are subjected to axial precompresion by placing loads ranging $0-750 \mathrm{~g}$.
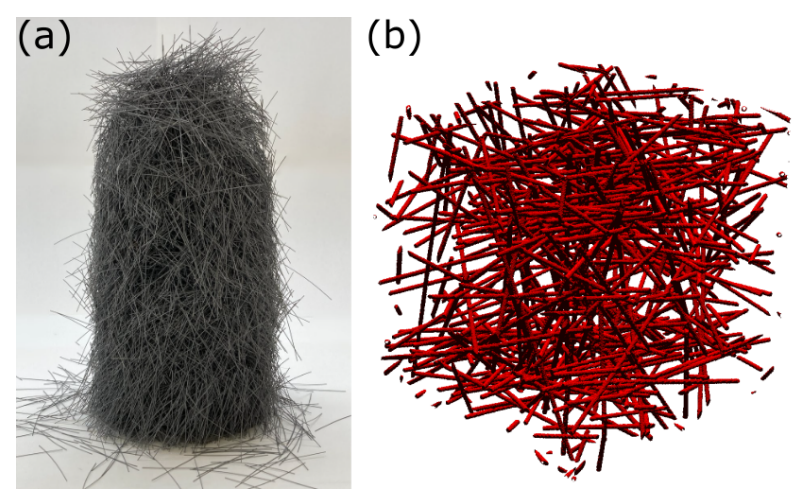

Figure 1. (a) Snapshot of a tower of fibres. (b) 3D reconstruction of a fibre assembly via X-ray microtomography.

**e-mail: ignacio.andrade-silva@univ-amu.godefroy@etu.univ-amu.fr

***e-mail: olivier.pouliquen@univ-amu.fr

****e-mail: joel.marthelot@univ-amu.fr

A video is available at https://doi.org/10.48448/q8r9-hf96

(c) The Authors, published by EDP Sciences. This is an open access article distributed under the terms of the Creative Commons Attribution License 4.0 

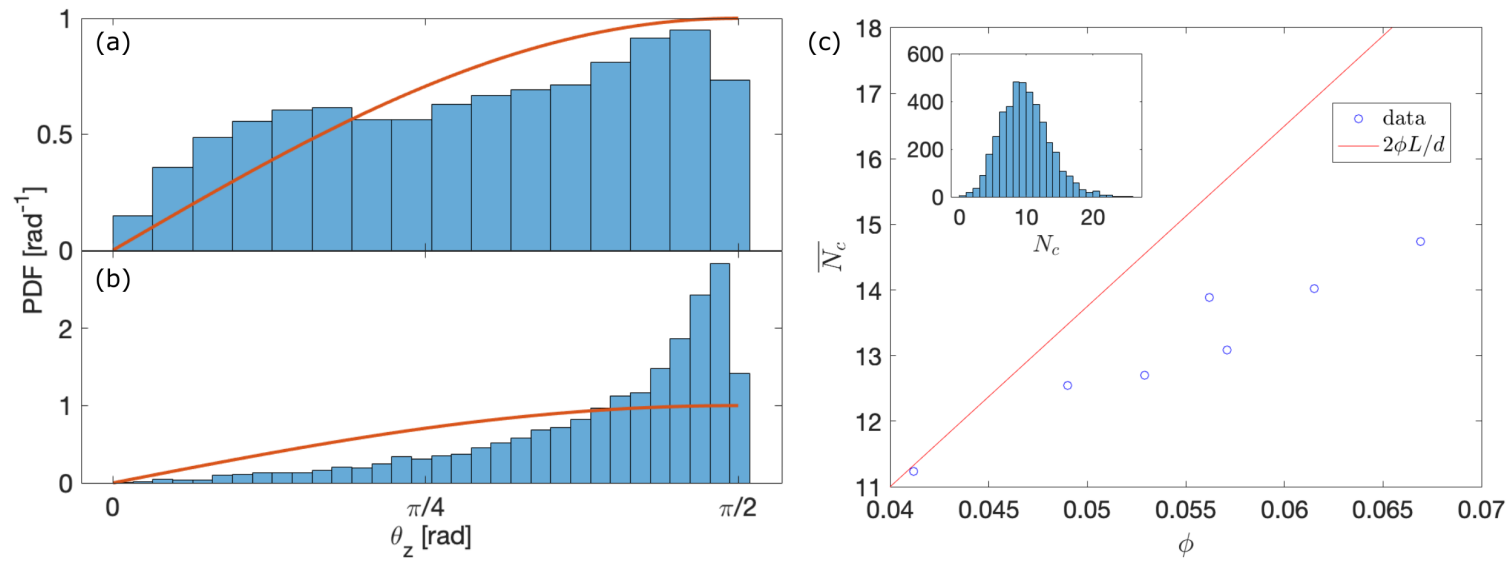

Figure 2. (a-b) Histograms of the angle $\theta_{z}$ of a fibre with respect the vertical axis of the column for a sample without pre-compresssion (a) and with $750 \mathrm{~g}$ load (b). The red orange curve represents the distribution $\sin \left(\theta_{z}\right.$ ) expected for a fully isotropic system. (c) Number of contacts as a function of the packing fraction. The red curve stands for the theoretical prediction $N_{c}=2 \phi L / d$ for an isotropic assembly. The inset show the distribution of number of contacts for a sample without precompression.

\subsection{X-ray microtomography and image processing}

We performed X-ray microtomography (voxel size of $71 \mu \mathrm{m})$ of the samples for several precompression loads. Fig. 1(b) shows the 3D reconstruction of a portion of a sample without load. We implement an image processing procedure that allows us to extract the centreline of each fibre in the assembly. First, the 3D images are treated using a Hessian filter (3) that enhances tubular structures of known diameter and then binarized. This process removes the contacts in the binary volume, allowing to identify individual fibres as isolated objects in the binary volume. Each object is then skeletonized and the skeletons are smoothed obtaining so the centrelines of all the fibres. The binarization step may cause that some detected fibres breaks into shorter segments, which is repaired in a subsequent step based on the local directions at the ends of the broken fibres. At the end of the process, we obtain the centrelines of all the fibres in the scanned volume with high accuracy, which give us access to valuable statistical information about fibre orientation, packing fraction, mean curvature and the average number of contact per fibre.

Figures 2(a-b) show the orientational distributions of the assembly at two different vertical loads, revealing a reorientation of the fibres towards the horizontal plane as the sample is compressed. The packing fraction $\phi$ of the sample is estimated by summing up total length of fibre centrelines contained in a cubic volume inside the scanned volume multiplied by cross section area of the fibres and then dividing this value by the volume of the cubic region. We also count the number of contacts in the volume by computing shorter distances between the centrelines of two fibres and determine contact if this distance is comparable to the diameter of the fibres. Fig. 2(c) shows the average number of contacts per fibre $\overline{N_{c}}$ as a function of $\phi$. For low precompression, the latter quantity is in good agreement with the formula $\overline{N_{c}}=2 \phi L / d$ proposed by Toll [5] for randomly oriented slender fibres. However, for fur- ther precompression the data deviates from the prediction probably due to the reorientation of fibres.

\section{Mechanical characterisation}

\subsection{Compression tests}

We first characterise the elastoplastic response in compression of the packed fibres. Fig. 3 shows a typical force-displacement curve of a fibre tower under several cycles of axial compression. The mechanical response is highly non-linear with large hysteresis and permanent compaction of the assembly, while the individual deformation of each fibre stays in the elastic regime. We characterise this macroscopic plastic regime and the influence of the packing fraction on the elastic response of the assembly.

Qualitatively, this curve is consistent with previous observations in textile materials [2,3], which are known to exhibit large hysteresis cycles, demonstrating a highly plastic response. We can relate the nonlinearity of the

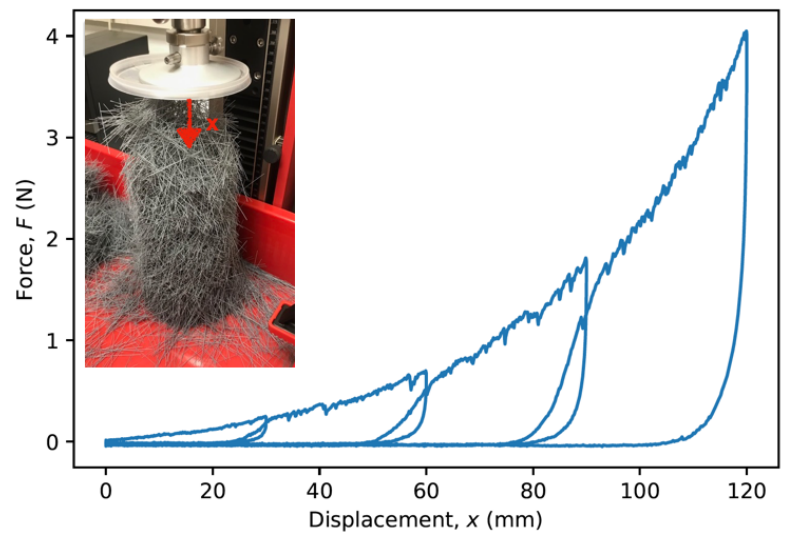

Figure 3. Typical force-displacement curve of axial compression cycles of a tower of fibres. 

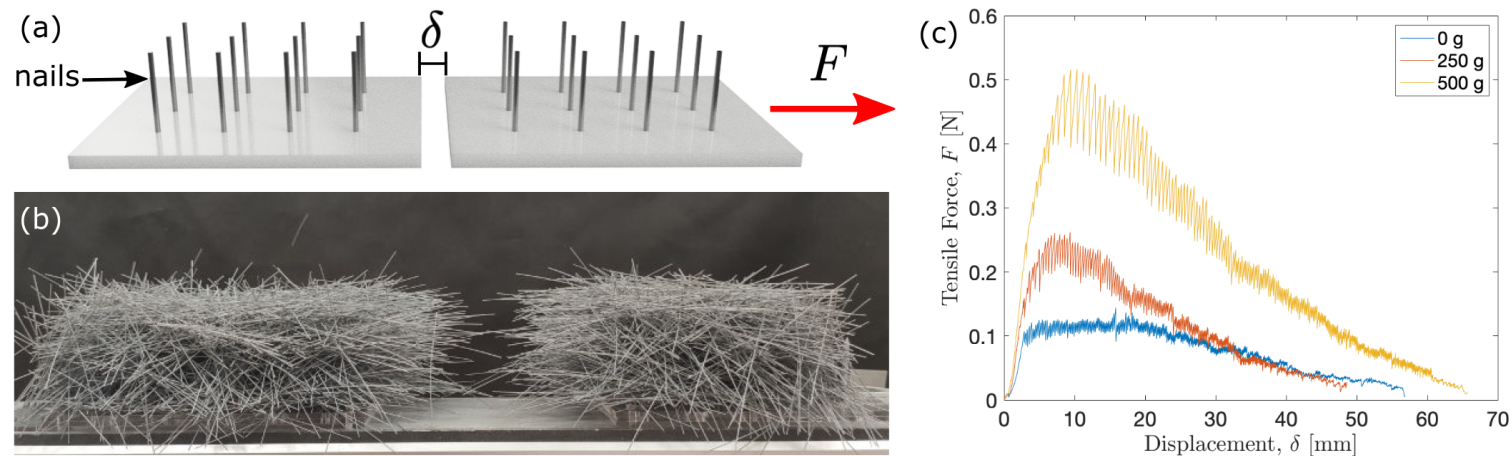

Figure 4. (a) Scheme with the double bed of nails setup used for the traction test. (b) Snapshot of a traction test after fracture. (c) Force-displacement curves of axial traction for 3 pre-compression loads.

force to the increasingly number of contacts and fibre reorientation throughout the compression test.

\subsection{Measuring cohesion}

To characterise the effective cohesive strength of a fibre assembly, one may think in subjecting the sample to a traction test and define the cohesive strength as the force necessary to fracture it. However, one cannot apply this idea in the same way as one would do with a single body solid. In the case of fibres, clamping or attaching two parts of the aggregate implies the introduction of stresses that disturbs the effective cohesion of the original state of the sample. To overcome this issue we propose an experimental protocol that minimises the influence of the way the sample is hold. To impose a traction displacement $\delta$ on the fibre assembly without perturbing the system, we use minimally invasive clamps by building directly the assembly on two horizontal acrylic plates $(10 \times 10 \mathrm{~cm}$ each $)$ with nails pointing upward (see Fig. 4(a)). The aggregate can be precompressed by putting a load on the top and then, the lateral walls of the box are removed. The two plates are connected to a horizontal universal testing machine to measure the stress-strain curve.

The test always ends up with the fracture of the aggregate in two parts, where one can clearly distinguish a region characterised by fibre realignment (process zone), while the regions outside remain with no noticeable deformation (Fig. 4(b)). We observe that the traction force increase to a maximum value and then decays to zero when the displacement $\delta$ reaches the fibre length (Fig. 4(c)). This maximum can be related to the effective cohesive strength of the aggregate. We show that this effective cohesive strength depends critically on the initial packing fraction of the assembly. Moreover, we image X-ray microtomography of the process zone for several values of $\delta$ to extract information about fibre reorientation and loss of contacts throughout traction to relate the macroscopic cohesive strength of the assembly with the filament reorganisation at the microscopic scale.

\section{Conclusion}

Although the mechanical response of random fibre assemblies under compression has been studied in the past, almost no studies focus one their effective cohesion. In the present study we proposed an experimental protocol that allows us to define the cohesive strength as the fracture force measured under traction with relatively no influence of boundary conditions. Moreover, by imaging the sample with in situ X-ray microtomography, we are able to obtain global or local statistical properties of the aggregates in either equilibrium or throughout traction tests to relate the macroscopic mechanical behaviour of the assembly with the fibres reorganisation at the microscopic scale.

\section{References}

[1] N. Weiner, Y. Bhosale, M. Gazzola, H. King, J. App. Phys. 127, 050902 (2020)

[2] R. C. Picu, Soft Matter 7, 6768-6785 (2011)

[3] C. M. Van Wyk, J. Text. Inst. 37, 12 (1946)

[4] A. F. Frangi, W. J. Niessen, K. L. Vincken, M. A. Viergever, Multiscale vessel enhancement filtering, in Medical Image Computing and Computer-Assisted Intervention - MICCAI'98, Lecture Notes in Computer Science 1496, 130-137 (1998)

[5] S. Toll, Polym. Eng. Sci. 38, 1337-1350 (1998) 